Special issue of the 3rd International Conference on Computational and Experimental Science and Engineering (ICCESEN 2016)

\title{
Wing Aerodynamic Optimization by Using Genetic Algoritm and Ansys
}

\author{
I. CAYiroglu ${ }^{a}$ And R. Kilic ${ }^{b, *}$ \\ ${ }^{a}$ Karabuk University, Mechatronic Engineering Department, Karabuk, Turkey \\ ${ }^{b}$ Sakarya University, Sakarya M.Y.O. 54187 Sakarya, Turkey
}

\begin{abstract}
The design of aircraft wings can be examined in two ways, namely, by aerodynamic analysis and by structural analysis. In aerodynamic terms, the wing is expected to display such features as maximum lifting load, minimum drag force, and high stall performance; in structural terms, it is desired to be light, robust, and away from vibration effects. In this paper optimization of the wing aerodynamic analysis of a private jet plane has been performed. Wing simulation was conducted with Ansys-Fluent program, whereas optimization of design criteria was realised using genetic algorithm. Design criteria determined in parametric terms have been optimized with genetic algorithm, which was written in Python, inside the Ansys-Workbench. Python was not sufficient on its own for the realization of the genetic algorithm and for control of the Ansys modules, as a result, it was assisted with Javascript and Journaling. The developed method can be used in a variety of design applications.
\end{abstract}

DOI: 10.12693/APhysPolA.132.981

PACS/topics: 47.85.Gj, 47.11.Fg

\section{Introduction}

Wings are the most critical elements in the structural systems of aircrafts. In addition to the requirement that they must have a thin projection, they carry the load of the entire structure. A well-designed wing must be evaluated using many design objectives and constraints, such as lift/drag ratio, stall performance, lightness and so forth [1].

Currently, the aerodynamic design is taking advantage of the rapid development of modern optimization methods [2-4]. Genetic algorithm [4-8] and adjoint method $[9,10]$ are two kinds of widely used optimization methods of airfoil design. Both methods have their inherent problems [11].

The genetic algorithm (GA) is a popular optimization method for engineering applications [12]. This algorithm is theoretically a global optimum searching method. It has good flexibility with regard to setting design constraints and objectives. The GA also has independence from the aerodynamic equations and can avoid complicated formulations. However, using entirely 3D wing optimization, based on the GA, to achieve a real globally optimal solution is still difficult because of the considerable computational cost. Computation cost of computational fluid dynamics (CFD) must be carefully controlled in GA optimization. "Man-in-loop" design process is a practical compromise for engineering applications [13]. In this study manual interventions were made to the optimization process occasionally.

Kumar et al. [14] performed the preliminary sizing and analysis of a training plane. Elham [15] presented

*corresponding author; e-mail: recep.kilic@sakarya.edu.tr a quasi-three-dimensional method for drag estimation for aerodynamic analysis of the wing. In their study, Zhang et al. [11] tested a supercritical natural-flow wing design using GA and CFD. Zhao et al. [1] developed an optimization method based on the pressure distribution transformation between wing profile and the wing for a supercritical wing design. They compared the presented $2.75 \mathrm{D}$ method with the $3 \mathrm{D}$ wing optimization.

In this paper, optimum aerodynamic design at subsonic speed of a plane wing has been performed using GA. The ratio of lifting force to frag force was determined as the basic objective function. In addition, as a wing with minimum bending momentum would be at a better point in terms of resistance, the location of pressure center on $z$ axis was added to the objective function. Wing-length of $10 \mathrm{~m}$ from the plane axis and cruise speed of $0.58 \mathrm{Ma}$ were taken as design constants.

\section{Design of genetic algorithm}

Genetic algorithm is one of the most popular algorithms which can perform optimization in a robust and able manner in complex and noisy search spaces $[16,17]$. However, it is an expensive optimization tool, which sometimes requires thousands of analyses simultaneously in order to achieve convergence in reaching of the results. From this perspective, when design is made with classical GA procedure, the number of produced genetic codes and therefore number of analysis surpasses thousands. For this reason, in order to shorten this period, the limitations which produce genetic code were chosen in a narrow area and with increasing values. Variables were connected to each other and production of useless genes was prevented.

\subsection{Optimization procedure}

In optimization process, one cycle of design process is realised in five stages. After the GA takes as starting 
values the design variables and constants received from the user, it produces design parameters (genes) based on standard genetic procedure (generation, evaluation, selection, crossover, mutation). These produced genes are automatically transformed into geometric solid model inside the Design Modeller and then they are meshed in the Mechanical Modeller, according to the given criteria. Later, through Fluent module, boundary conditions are implemented and analysis results are recorded in the file. The obtained design results are read and evaluated by Python code; if they are not adequate, analysis process is restarted with the production of another gene. All these processes are automatically performed in a cycle with the help of a script (Fig. 1).

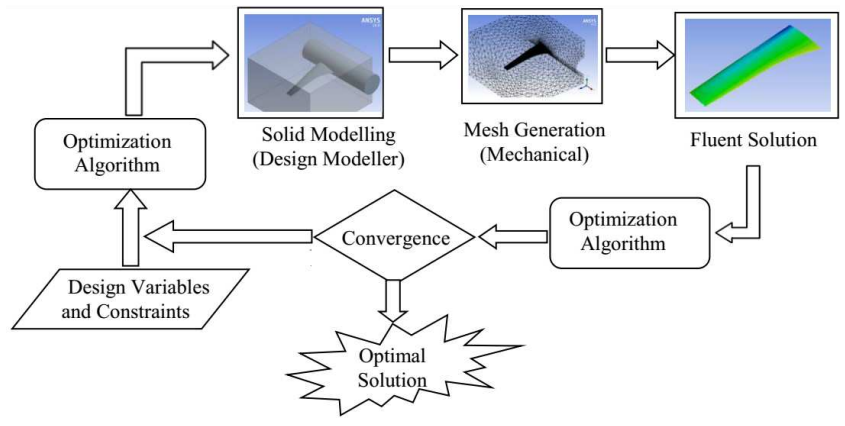

Fig. 1. Optimization procedure.

\subsection{Objective function}

The most important design criteria for an aircraft wing in terms of aerodynamics are maximum lift and minimum drag force. However, these two criteria are not sufficient for meeting all design expectations. As the structural optimization of the wing is not performed, parameters which will meet relevant expectations must also be included in the design criteria. For this purpose, the distance of pressure center of the wing to the wing root is added as well. Thus, an effort is made to reach the minimum pitching moment value. In order to achieve this, the root of the wing must be wide and the tip of the wing must be narrow. In addition, in order to decrease pitching moment, relative lifting force must be decreased towards the ends. For this purpose, changes were made to the wing angle of attack and to profile shape. As a result, design criteria were determined as (lift/drag) $\max$ and (pitching moment) $\min$.

\subsection{Design variables}

Aerodynamic design variables were examined in two groups. The first group contained NACA parameters which had formed the profile cross-sections of wing, and the second group consisted of the parameters which give the wing its shape (Fig. 2). The shape of the NACA airfoils is described using a series of digits following the word "NACA". The parameters in the numerical code can be entered into equations to precisely generate the crosssection of the airfoil and calculate its properties.

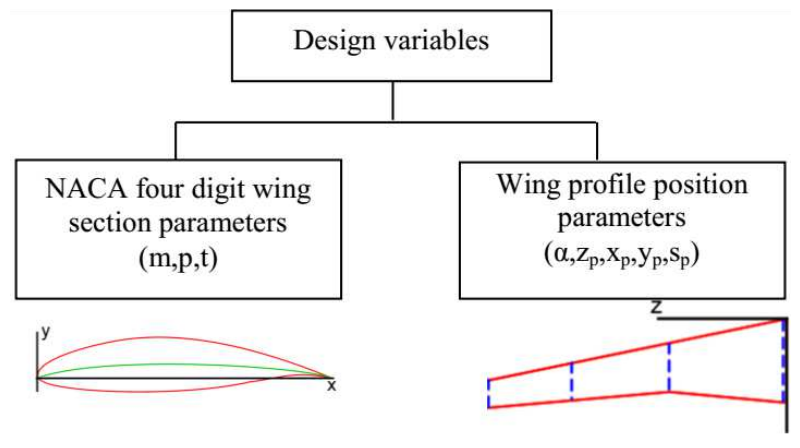

Fig. 2. Design variables.

Profile cross-sections were produced with NACA 4-digit series. For example, the NACA 2412 airfoil has a maximum camber of $2 \%$ located $40 \%$ ( 0.4 chords) from the leading edge with a maximum thickness of $12 \%$ of the chord.

The equations are:

$$
\begin{aligned}
x & =1-\cos \left(n_{i} \frac{90}{n-1}\right), \\
y_{t} & =\frac{t}{0.2}\left(0.2969 \sqrt{x}-0.1260 x-0.3516 x^{2}\right. \\
& \left.+0.2843 x^{3}-0.1015 x^{4}\right), \\
y_{c} & = \begin{cases}\frac{m}{p^{2}}\left(2 p x-x^{2}\right) & \text { for } x<p, \\
\frac{m}{(1-p)^{2}}\left((1-2 p)+2 p x-x^{2}\right) & \text { for } \quad x>p .\end{cases}
\end{aligned}
$$

Here $t$ is the maximum thickness as a percentage of the chord, $m$ is the maximum camber as a percentage of the chord, $p$ is the chordwise position of the maximum camber, as a tenth of the chord (Fig. 3). Equation (1) shows the coordinate locations on axis $x$. The scatter is more frequent in front of the profile but it is more sparse in the back. $n$ shows the number of points with which the profile will be formed.
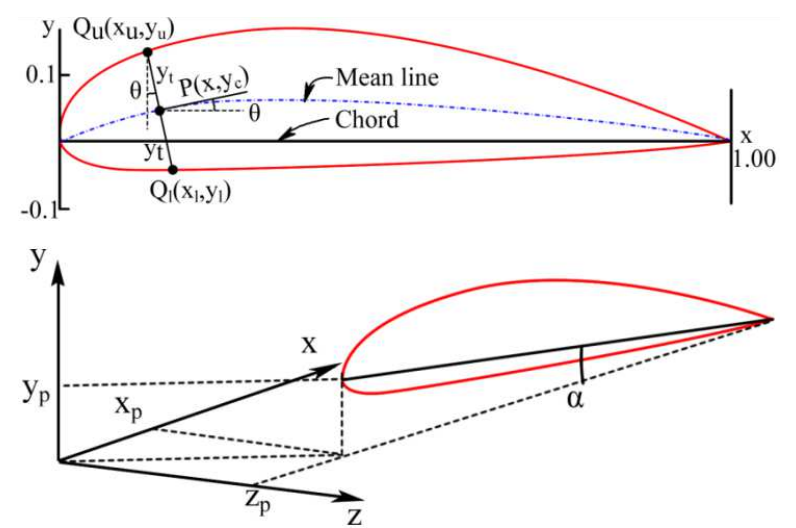

Fig. 3. NACA four-digit wing section and position parameters.

The complete geometry is given by

$$
\begin{aligned}
& x_{u}=x-y_{t} \sin \theta, \quad y_{u}=x+y_{t} \cos \theta, \\
& x_{l}=x+y_{t} \sin \theta, \quad y_{l}=x-y_{t} \cos \theta,
\end{aligned}
$$


The wing has consisted of four profile cross-sections. NACA parameters which form the shape of these profiles and the location parameters of the profile, which forms the shape of the wing, are given in Table I, collectively.

TABLE I

Optimization design variables.

\begin{tabular}{c|c|l}
\hline \hline No & Variable & Definition \\
\hline 1 & $m$ & $\begin{array}{l}\text { Maximum camber percentage of the chord } \\
2\end{array}$ \\
3 & $t$ & $\begin{array}{l}\text { Chordwise position of the maximum cam- } \\
\text { ber as a tenth of the chord } \\
\text { Maximum thickness percentage of the } \\
\text { chord }\end{array}$ \\
4 & $\alpha$ & Angle of attack \\
5 & $z_{\mathrm{p}}$ & $z$-axis offset position of profile \\
6 & $x_{\mathrm{p}}$ & $x$-axis offset position of profile \\
7 & $y_{\mathrm{p}}$ & $y$-axis offset position of profile \\
8 & $s$ & Profile scale factor
\end{tabular}

\subsection{Design constrains}

Optimized wing model was taken to be close to the wing size of small-size jet planes (10 $\mathrm{m}$ from axis). Depending on the navigation speed of the plane, the optimal aerodynamic form of the wing changes. Wing profile shape of the plane during stall speed will not be the same as the profile shape during cruising speed. At low speeds, camber level has to be increased, so as to increase lifting force. In real life slats and flaps are used in the leading edge and the trailing edge, respectively, so as to achieve this adjustment depending on speed. In this paper a constant-edge wing will be designed. As a result, a suitable navigation speed must be chosen. For this purpose speed was chosen as $720 \mathrm{~km} / \mathrm{h}(200 \mathrm{~m} / \mathrm{s}, 0.58 \mathrm{Ma})$.

While assigning design parameters, rather than choosing them randomly, the parameters of each profile, which constitute the wing, were assigned in such way, as to ensure certain constraints, depending on the previous profile, starting from the root. Thus, production of unnecessary models which will deform the shape of the wing, was prevented. The used constraints are given in Table II.

TABLE II

Optimization design constraints of profiles (index no. 4 corresponds to wing-tip and index no. 1 corresponds to wing-root).

\begin{tabular}{c|c|c}
\hline \hline No & Constraint & Definition \\
\hline 1 & $8 \% \geq m_{4} \geq m_{3} \geq m_{2} \geq m_{1} \geq 0 \%$ & Camber percentage \\
2 & $70 \% \geq p_{4} \geq p_{3} \geq p_{2} \geq p_{1} \geq 30 \%$ & Chordwise position \\
3 & $4 \% \leq t_{4} \leq t_{3} \leq t_{2} \leq t_{1} \leq 20 \%$ & Maximum thickness \\
4 & $0^{\circ} \leq \alpha_{4} \leq \alpha_{3} \leq \alpha_{2} \leq \alpha_{1} \leq-10^{\circ}$ & Angle of attack \\
5 & $z_{\mathrm{p} 4}=10 \mathrm{~m} \geq z_{\mathrm{p} 3} \geq z_{\mathrm{p} 2} \geq z_{\mathrm{p} 1}=0 \mathrm{~m}$ & $z$-axis offset \\
6 & $6 \mathrm{~m} \geq x_{\mathrm{p} 4} \geq x_{\mathrm{p} 3} \geq x_{\mathrm{p} 2} \geq x_{\mathrm{p} 1}=0 \mathrm{~m}$ & $x$-axis offset \\
7 & $1 \mathrm{~m} \geq y_{\mathrm{p} 4} \geq y_{\mathrm{p} 3} \geq y_{\mathrm{p} 2} \geq y_{\mathrm{p} 1}=0 \mathrm{~m}$ & $y$-axis offset \\
8 & $0.2 \leq s_{4} \leq s_{3} \leq s_{2} \leq s_{1} \leq 1$ & Profile scale
\end{tabular}

\subsection{Chromosome encoding and genetic process}

In order to solve the optimization problems by means of GA, design variables must be coded into a list of genes (chromosome) and a design example must correspond to a chromosome that represents an individual. The complexity of an organism can be controlled by the length and number of chromosomes.

The shape of the wing consists of four profiles. The position parameters of each profile are created depending on the previous profile (Table II). Thus, formation of erroneous wing shapes was prevented.

Each gene string, forming each profile, is represented with eight genes in the chromosome, which forms the wing. Three of these genes determine the shape of NACA profile $(m, p, t)$ whereas the remaining five determine the position of profiles $\left(\alpha, z_{\mathrm{p}}, x_{\mathrm{p}}, y_{\mathrm{p}}, s_{\mathrm{p}}\right)$. In cross-over procedures, mutual gene strings were replaced with each other. The produced gene string was in accordance with the determined constraints. Mutation process was also performed once on a single gene in the production of each generation (Fig. 4).

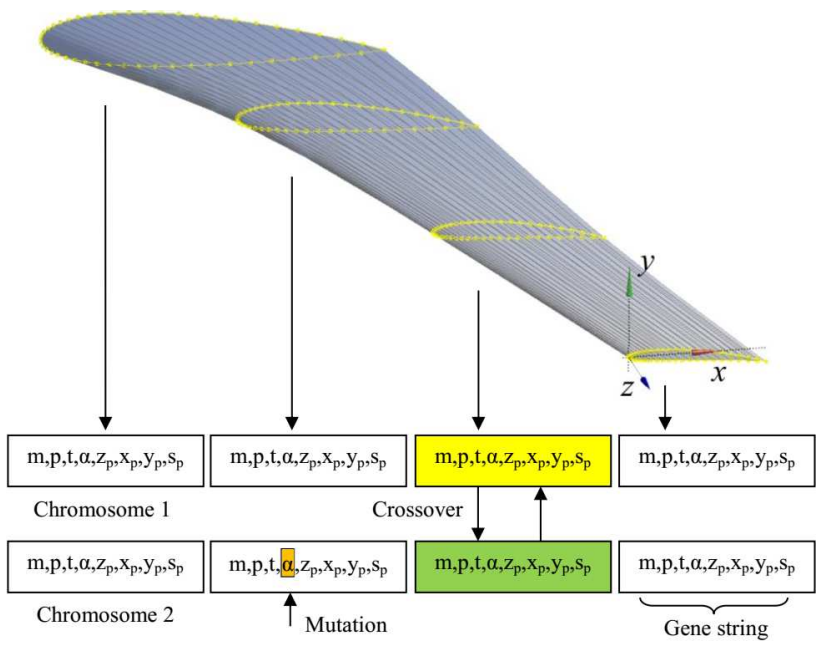

Fig. 4. Gene structure and genetic operations.

As fitness value, the three values, obtained from analyses (lift force, drag force, center of pressure), were used. These values were normalized to value of 20 (Eq. (6)) and multiplied by their weights, which gave the fitness value. As a result, the impact of $F_{\text {lift }} / F_{\text {drag }}$ ratio, showing the aerodynamic efficiency of the wing, which is aimed to be maximum, and the place of center of pressure $C_{\mathrm{pz}}$ on axis $z$, which is desired to be minimum for bending resistance of the wing, became $70 \%$ and $30 \%$ respectively in the fitness value.

$$
\begin{aligned}
& \text { Fitness }=\left[0.7 \frac{F_{\text {lift }}}{F_{\text {drag }}}\left(\frac{20}{\operatorname{Av} \cdot\left(\sum\left(\frac{F_{\text {lift }}}{F_{\text {drag }}}\right)\right)}\right)\right. \\
& \left.-0.3 C_{\mathrm{pz}}\left(\frac{20}{\operatorname{Av} \cdot\left(\sum C_{\mathrm{pz}}\right)}\right)\right] 2 .
\end{aligned}
$$


Twenty chromosomes were used in the first produced population and in the following populations of the genetic cycle. As analysis took too much time, the number of population was kept minimum. Tournament method was used for selection operation. Child genes produced after crossover and mutation processes were also subjected to fitness evaluation. Then, 10 good genes were chosen among the 20 genes and 10 more genes were produced randomly to create a new parent generation. This new generation was subjected to the same genetic procedures and optimization continued until the stop criterion was ensured (Fig. 5). The following criterion was used as the stop criterion: if a better wing model could not be found throughout five cycles, the program would be stopped.

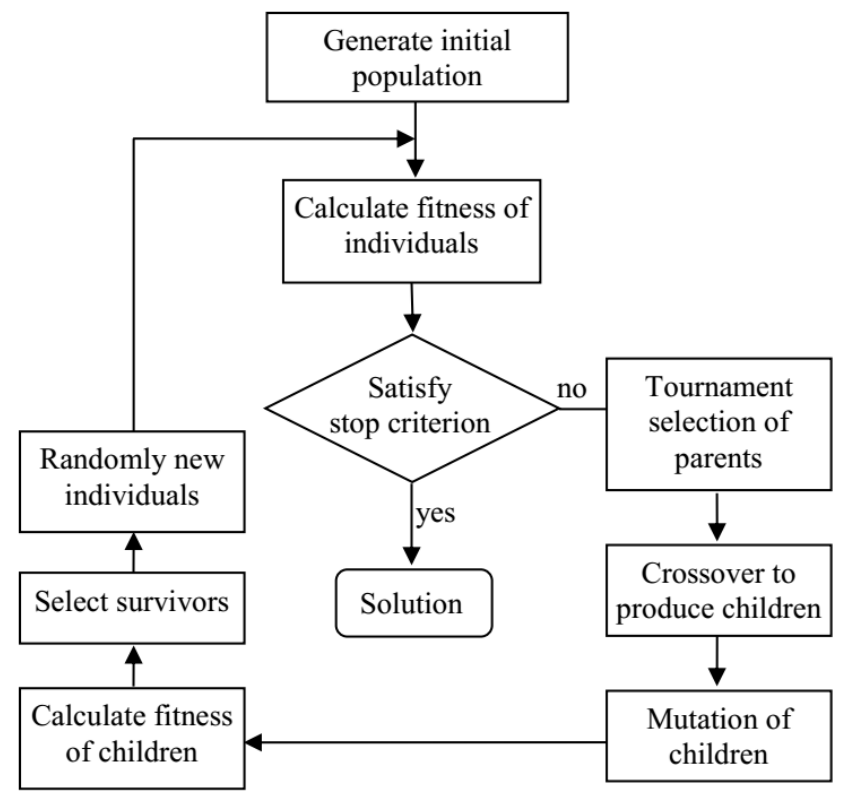

Fig. 5. GA flow chart.

\section{Finite element model}

\subsection{Ansys scripting}

In general, most of the Ansys tools are "scriptable". Ansys Workbench uses Python as script language, whereas Ansys Design Modeller uses Javascript. In this paper both languages were used. Javascript code was used to create profile sketches, and Python code was used to create solid model of the wing. In addition, the codes of "Journaling", which are the recording coding of Ansys, were created in order to perform fluent procedures, which were embedded in Python code. The entire coding was operated inside Workbench.

While the procedures were being performed automatically, manual intervention was needed in certain occasions. For example, while creating Loft command in some complex models, distorted shapes were created due to the erroneous positioning of the "Guide Line". In such cases manual intervention made sure that the guide line was located accurately.

\subsection{Meshing and boundary conditions}

As solid model, the model of the air outside the wing surface was subjected to analysis. Boundary conditions at the front surface from which the air enters, at the rear surface from which the air exits, at hull and wing surfaces, and at sections for passing from air to air were determined as "velocity inlet", "pressure outlet", "wall", and "symmetry", respectively. An average of $600000-$ node meshes were used in solid model. The smallest mesh size was taken as $50 \mathrm{~mm}$. At this mesh density the model converged in some 15 minutes, at an average of 200 iterations. When wing profiles were created with spline command, the mesh operation at trailing edge had usually produced an error. As a result, four profiles, which constitute the wing were created with 80 flat lines and the wing shape was produced by making Loft among them (Fig. 6).
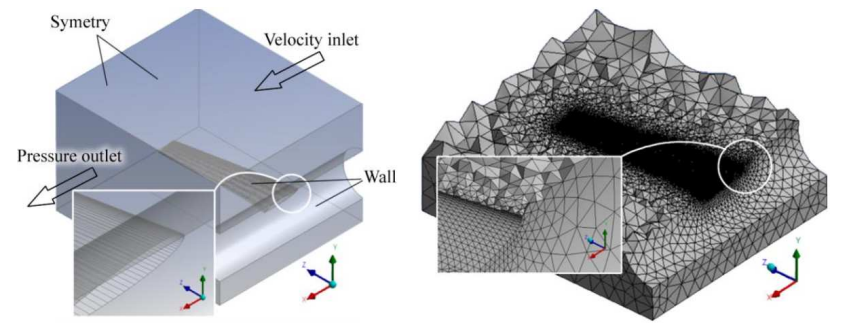

Fig. 6. Boundary conditions in solid model and mesh structure.

\section{Discussion and conclusions}

Fitness value results obtained according to Eq. (6), as a result of 36 genetic cycles, are given in Fig. 7a. Lift/drag results, which show the aerodynamic efficiency of the wing, which affects this graphic, are given in Fig. 7b and the graphic showing the change in center of pressure is given in Fig. 7c. Figure. 7d shows the plot of Lift/drag vs center of pressure. When these graphs are examined, it can be seen that results began to converge after some 16 cycles. The wing with the best features is the one with the highest Lift/drag ratio and the lowest center of pressure distance.

Pressure distribution around the best wing, obtained as a result of genetic optimization, is given in Fig. 8 . The highest pressure on lower surface of the wing and the lowest pressure on the upper surface were $19200 \mathrm{~Pa}$ and $-20100 \mathrm{~Pa}$, respectively. While the wing travels in the air with the speed of $720 \mathrm{~km} / \mathrm{h}$, the lifting and drag forces are $158324 \mathrm{~N}$ and $4313 \mathrm{~N}$, respectively. These forces include the viscous forces. The $k$-epsilon model was used as the turbulence model.

The four NACA profiles which gave the wing its shape were NACA1414, NACA2309, NACA2408 and NACA2407. The point of origin of each profile was located at $0,857,1714,4252 \mathrm{~mm}$ on axis $x ; 0,200,300$, $300 \mathrm{~mm}$ on axis $y$ and $0,2000,4000,10000 \mathrm{~mm}$ on axis $z$, 


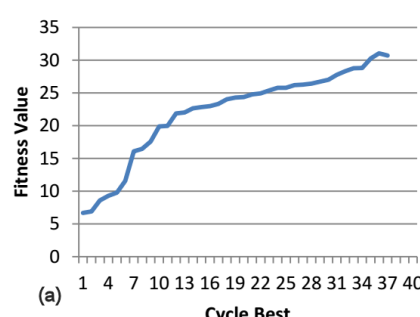

Cycle Best
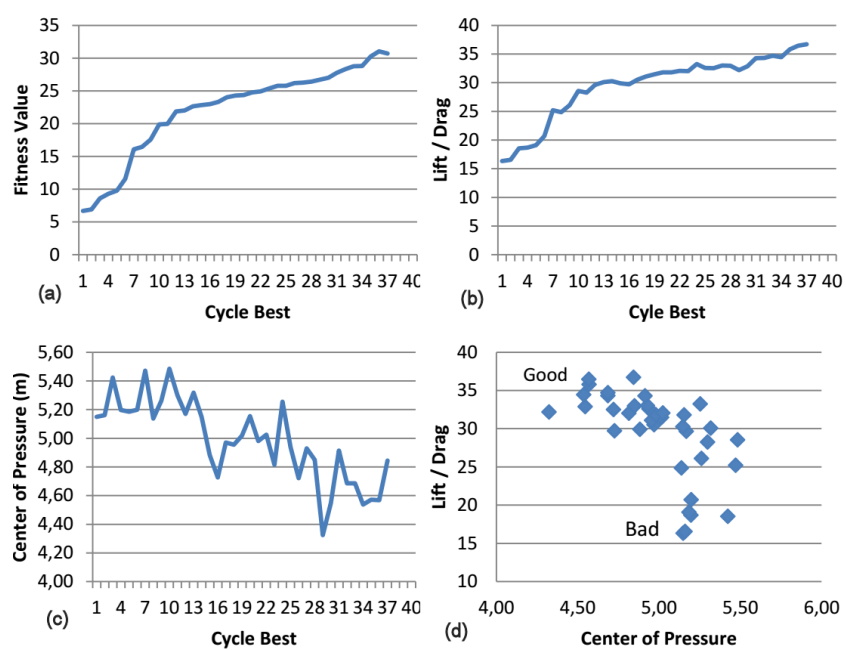

Fig. 7. Per cycle results of genetic algorithm.
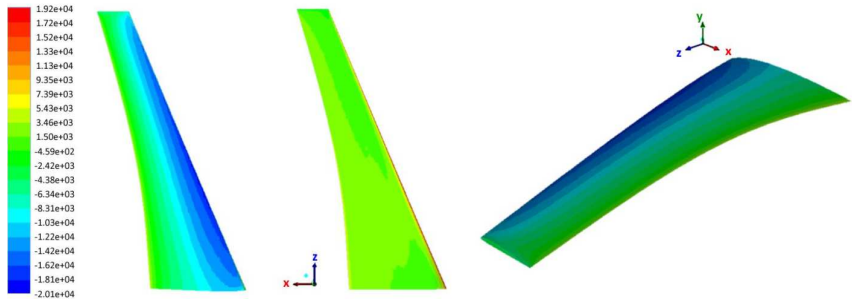

Fig. 8. Pressure distribution on the best wing model obtained as a result of 36 optimizations.

according to the global wing axes. Angles of attack were $-4^{\circ},-3^{\circ},-2^{\circ}$ and $-2^{\circ}$, whereas profile lengths were 3498 , 2802, 2106 and $984 \mathrm{~mm}$. Table III gives the parameters which consist of the aerodynamic features of final design.

TABLE III

Parametric features of the wing obtained using optimization.

\begin{tabular}{l|c}
\hline \hline Details & Profile results \\
\hline $\begin{array}{l}\text { Maximum camber percentage of } \\
\text { the chord [\%] }\end{array}$ & $1,2,2,2$ \\
$\begin{array}{l}\text { Chordwise position of the maxi- } \\
\text { mum camber, as a tenth of the } \\
\text { chord }\end{array}$ & $4,4,4,4$ \\
$\begin{array}{l}\text { Maximum thickness percentage } \\
\text { of the chord [\%] }\end{array}$ & $14,9,8,7$ \\
$\begin{array}{l}\text { Angles of attack [degrees] } \\
\text { Profile lengths [mm] } \\
z \text { offset [mm] }\end{array}$ & $-5,-4,-4,-2$ \\
$x$ offset [mm] & $3498,2802,2106,984$ \\
$y$ offset [mm] & $0,2000,4000,10000$ \\
& $0,857,1714,4252$ \\
& $0,200,300,300$
\end{tabular}

This developed method can be used in several design processes. The point here is that the control of Ansys is performed with limitless cycles and the design parameters are entered using a script while the results are also read by a script. As analysis takes too much time, the design variables are determined inside narrow limits and incremental values in line with expectations. After optimum wing is determined, a more sensitive research can be conducted around the obtained parameters and wing characteristics can be improved further.

\section{References}

[1] T. Zhao, Y. Zhang, H. Chen, Y. Chen, M. Zhang, Aerospace Sci. Technol. 56, 168 (2016).

[2] A. Jameson, S. Kim, AIAA J. 41, 2114 (2003).

[3] A. Jameson, S. Shankaran, L. Martinelli, AIAA J. 46, 1226 (2008).

[4] Y. Zhang, X. Fang, H. Chen, S. Fu, Z. Duan, Y. Zhang, Aerospace Sci. Technol. 43, 152 (2015).

[5] Y. Zhang, H. Chen, W. Zhang, S. Fu, M. Zhang, M. Zhang, T. Liu, in: 50th AIAA Aerospace Sciences Meeting, 2012, p. 1046.

[6] A.P. Antunes, J.L.F. Azevedo, R.G. Silva, in: 47th AIAA Aerospace Sciences Meeting, 2009, p. 1094.

[7] T. Zhao, Y. Zhang, H. Chen, in: 53rd AIAA Aerospace Sciences Meeting, 2015, p. 0763.

[8] A. Nobakhti, Cogn. Comput. 2, 97 (2010).

[9] J.D. Lee, A. Jameson, in: 47th AIAA Aerospace Sciences Meeting, 2009, p. 897.

[10] J.Q. Luo, J.T. Xiong, F. Liu, Phys. Mech. Astron. 57, 1363 (2014).

[11] Y. Zhang, X. Fang, H. Chen, S. Fu, Z. Duan, Y. Zhang, Aerospace Sci. Technol. 43, 152 (2015).

[12] K. Deb, A. Pratap, S. Agarwal, T. Meyarivan, IEEE Trans. Evolutionary Computat. 6, 182 (2002).

[13] Y. Zhang, H. Chen, M. Zhang, M. Zhang, T. Liu, W. Zhang, S. Fu, in: 49th AIAA Aerospace Sciences Meeting, 2011, p. 27.

[14] T.S.V. Kumar, A.W. Basha, M. Pavithra, V. Srilekha, Int. J. Res. Aeronautical Mech. Eng. 3, 1 (2015).

[15] A. Elham, Aerospace Sci. Technol. 41, 241 (2015).

[16] M.K. Cakir, Ms. Sci. Thesis, Graduate School of Natural and Applied Sciences of Middle East Technical University, 2008.

[17] Y. Hei, W. Li, M. Li, Z. Qiu, W. Fu, Cogn. Comput. 7, 359 (2015). 